(C) 2017, THE AUTHORS. Published by FASS and Elsevier Inc. on behalf of the American Dairy Science Association ${ }^{\circledR}$.

This is an open access article under the CC BY-NC-ND license (http://creativecommons.org/licenses/by-nc-nd/3.0/).

\title{
Lower working heights decrease contraction intensity of shoulder muscles in a herringbone $30^{\circ}$ milking parlor
}

\author{
Marianne Cockburn, ${ }^{* 1}$ Matthias Schick, ${ }^{*}$ Nicola A. Maffiuletti, $†$ Lorenz Gygax, $\ddagger$ Pascal Savary, ${ }^{*}$ \\ and Christina Umstätter* \\ *Agroscope Tänikon Federal Research Station, Tänikon 1, 8356 Ettenhausen, Switzerland \\ †Human Performance Lab, Schulthess Klinik Lengghalde, 8008 Zürich, Switzerland \\ †Centre for Proper Housing of Ruminants and Pigs, Federal Food Safety and Veterinary Office FSVO, Agroscope, \\ Institute of Livestock Sciences ILS, Tänikon 1, 8356 Ettenhausen, Switzerland
}

\begin{abstract}
Musculoskeletal disorders have been a main concern in milkers for many years. To improve posture, a formula was developed in a previous study to calculate ergonomically optimal working heights for various milking parlor types. However, the working height recommendations based on the formula for the herringbone $30^{\circ}$ parlor were broad. To clarify the recommendations for the optimal working height, we investigated the effect of working height on upper limb and shoulder muscle contraction intensities. We evaluated 60 milking cluster attachment procedures in a herringbone $30^{\circ}$ milking parlor in 7 men and 9 women. Specifically, we examined the effect of working height on muscle contraction intensity of 4 arm and shoulder muscles bilaterally (flexor carpi ulnaris, biceps brachii, deltoideus anterior, and upper trapezius) by using surface electromyography. The working heights (low, medium, and high), which reflect the ratio of the subject's height to the height of the udder base, were used in the milking health formula to determine and fit individual depth of pits. Data were evaluated for each muscle and arm side in the functions holding and attaching. Statistical analysis was performed using linear mixed effects models, where muscle contraction intensity served as a target variable, whereas working height coefficient, sex, subject height, and repetition were treated as fixed effects, and repetition group nested in working height nested in subject was considered a random effect. Contraction intensities decreased with decreasing working height for the deltoideus anterior and upper trapezius, but not for the flexor carpi ulnaris or the
\end{abstract}

Received June 16, 2016.

Accepted January 30, 2017.

${ }^{1}$ Corresponding author: marianne.cockburn@agroscope.admin.ch biceps brachii muscles in both holding and attaching arm functions. We found that milking at a lower working height reduced muscle contraction intensities of the shoulder muscles. Women showed higher contraction intensities than men, whereas subject height had no effect. The study demonstrated that a lower working height decreased muscular load during milking. These lower working heights should be used within the recommendations made by the milking health formula for the herringbone $30^{\circ}$. Working heights could be adjusted effectively for milkers of varying body height. Future studies should therefore use the milking health formula as a tool to objectively compare and improve the accuracy of the working height coefficients.

Key words: milking health formula, ergonomics, physical load, electromyography

\section{INTRODUCTION}

Milking parlor productivity is extremely important, yet human health should not be neglected in this environment. Due to ongoing reports of high levels of musculoskeletal problems in milking parlor operators (Jakob, 2010; Patil et al., 2012; Douphrate et al., $2013,2014)$, research has aimed to evaluate risk factors during milking, such as posture (Jakob et al., 2012; Cockburn et al., 2015; Jakob and Thinius, 2015) and physical load (Liebers et al., 2009). A recent European study reported that $84 \%$ of Swedish and $85 \%$ of German milkers that work in milking parlors suffered from complaints in one or more body parts (Lunner Kolstrup and Jakob, 2016). Milking personnel has generally been advised to work in a position in which the teat ends are at shoulder level (Jakob et al., 2009, 2012). Liebers et al. (2009) monitored 6 female milkers using surface electromyography and found that light clusters could reduce muscle contraction intensities. Further, they concluded that working at shoulder height would decrease muscular load (Liebers et al., 2009). Contrarily, 
in a recent study, we found that raising the floor level for the milker would decrease the lifting height and, thus, benefit posture (Cockburn et al., 2015). In the same study, we also provided recommendations regarding differing working heights (WH) for a variety of parlor types (autotandem, herringbone $30^{\circ}$, herringbone $50^{\circ}$, parallel, and rotary) and developed guidelines that were implemented in the milking health formula (Cockburn et al., 2015). These recommendations enabled the calculation of $\mathrm{WH}$ under consideration of the parlor type, the cows' mean udder height, and the milkers body height.

The WH recommendations provided by the milking health formula resulted in considerably lower $\mathrm{WH}$ than those currently used in a commercial setting, especially for herringbone $30^{\circ}$ and side-by-side parlors (Cockburn et al., 2015). Liebers et al. (2009) recommended a WH where the udder is at shoulder level; considering a median male milker of $1.75 \mathrm{~m}$ in height with a shoulder height of $1.45 \mathrm{~m}$, this results in a WH coefficient of 0.8 (Lange and Windel, 2013). Furthermore, the recommended $\mathrm{WH}$ coefficient for the herringbone $30^{\circ}$ parlor was very broad (between 0.7 and 0.9 ). As, for example, $53 \%$ of German farms and $30 \%$ of Swedish farms use herringbone parlors (Lunner Kolstrup and Jakob, 2016), it was valuable to gain further information on the correct working heights for this milking parlor type. The lower WH recommended by the milking health formula could be favorable not only in improving posture, but also for reducing muscle contraction intensity and, thus, the physical demand during milking. Therefore, additional information was needed to refine the recommended WH coefficients that had been derived from on-farm experiments for this milking parlor type. Muscular contraction intensities give advanced insight into the effect of physical procedures on the musculoskeletal system. Surface electromyography (sEMG) is commonly used to measure these contraction intensities. It has been suggested that static $(>10 \mathrm{~s})$ contraction intensities over $5 \%$ of the maximum voluntary contraction (MVC) must be avoided, and mean contraction intensities should remain below $10 \%$ but must remain below $14 \%$ of the MVC to prevent long-term damage or injuries (Jonsson, 1978).

The present study aimed to investigate the effect of WH on upper limb and shoulder physical workload by evaluating muscle contraction intensities during the milking procedure. We further expected to improve the precision of the milking health formula's optimal $\mathrm{WH}$ coefficient for the herringbone $30^{\circ}$ parlor in a laboratory setting. A secondary aim of our study was to investigate the milking health formula's ability to set comparable WH for subjects of different body heights.

\section{MATERIALS AND METHODS}

\section{Experimental Setting}

The study was carried out in the experimental milking parlor of Agroscope in Tänikon, Switzerland. This experimental milking parlor was a $2 \times 5$ herringbone $30^{\circ}$ parlor with a manure curb of $0.1 \mathrm{~m}$ (Figure 1; GEA Farm Technologies GmbH, Bönen, Germany). It was equipped with an adjustable floor and the clusterpositioning arm Posilactor (GEA Farm Technologies $\mathrm{GmbH}$ ), which allowed for a variation of $\mathrm{WH}$ and a steady positioning of the milking clusters. Cluster-positioning arms were used because they help standardize and smoothen the movements between subjects. The milking clusters used were the GEA "Classic 300" (GEA Farm Technologies $\mathrm{GmbH}$ ) and weighed $2.6 \mathrm{~kg}$. The short milk tubes were made of silicon, which improves handling (Siliconform, Türkheim, Germany).

Subjects were monitored while attaching a milking cluster to an artificial udder (IC KUH, Bad Bentheim, Germany). This udder was placed in a self-constructed wooden stand with an udder base height of $0.55 \mathrm{~m}$ (Figure 2). The wooden stand was equipped with trueto-scale hind legs with size proportions that reflected the mean size of cow legs at our research farm. The artificial udders were positioned in the middle of the left and right sides of the milking parlor (milking stalls 3 and 8 , Figure 1).

\section{Subjects}

The study was registered with the Swiss Ethics Commission of the Canton of Thurgau, Switzerland. Seven men and 9 women with milking experience, but without a daily routine, participated in the trial. Milkers without work routine were chosen to exclude habituated work procedures and, thus, be able to instruct a particular milking cluster attachment technique. All milkers were in good health, had a body mass index below 30 , and participated voluntarily. Women measured between 1.68 and $1.89 \mathrm{~m}$ (mean $1.76 \pm 0.06 \mathrm{~m}$ ) and men measured between 1.74 and $1.89 \mathrm{~m}$ (mean $1.81 \pm$ $0.05 \mathrm{~m}$ ) in height.

\section{Working Heights}

Three WH were individually installed for each subject (Figure 3). These were determined by using WH coefficients, which reflect the ratio of the height of the udder base + depth of pit to the height of the milker (equation 1): 
Left

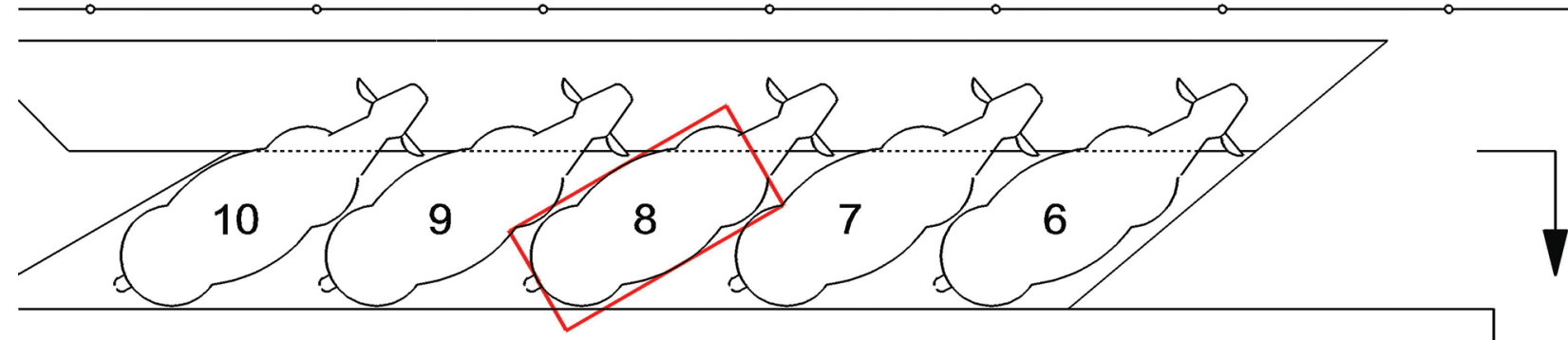

Milking pit

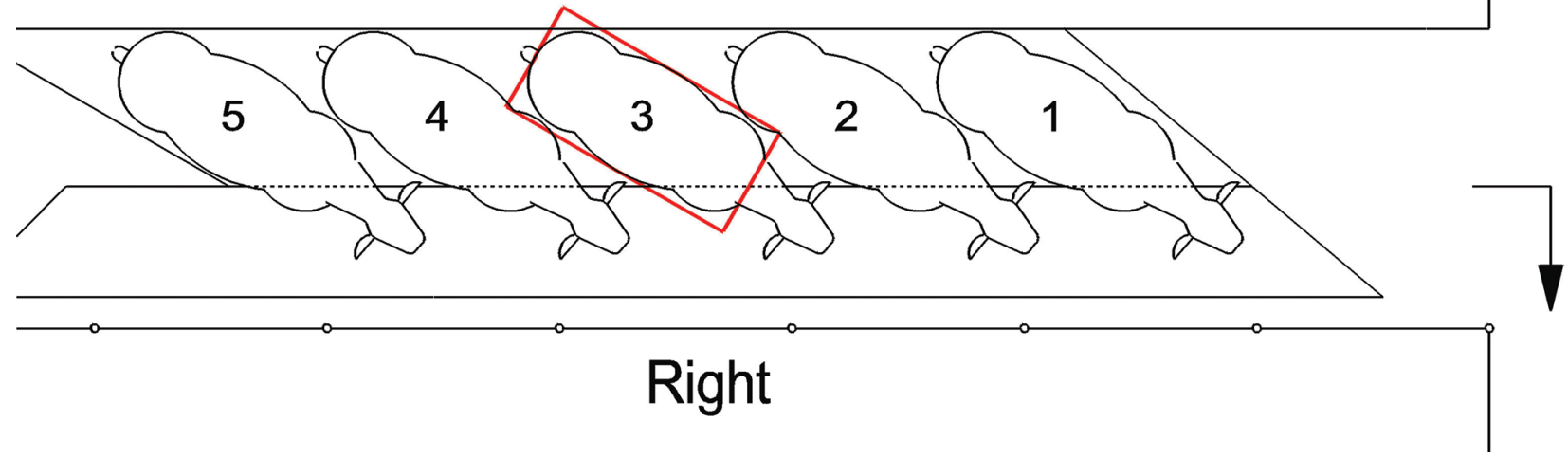

Figure 1. Setup of the experimental milking parlor. Two artificial udders were positioned at milking stall numbers 3 and 8 (outlined). The arrows indicate the cows' exit. Color version available online.

Depth of pit $(\mathrm{m})=[$ subject height $(\mathrm{m}) \times$ coefficient $]$

$$
-[\text { herd mean udder height }(\mathrm{m})] \text {, }
$$

where the coefficient is calculated as

$$
\begin{gathered}
\text { Coefficient }=[\text { udder base height }(\mathrm{m}) \\
+ \text { depth of pit }(\mathrm{m})] / \text { subject height }(\mathrm{m}) .
\end{gathered}
$$

The milking health formula (Cockburn et al., 2015) was used to calculate the individual depth of pit to ensure the correct WH (equation 1). In the formula, the WH coefficient was set as a constant factor and the height of the artificial udder $(0.55 \mathrm{~m})$ agreed with the herds' mean udder height. The WH coefficients 0.72 (low), 0.775 (medium), and 0.82 (high) were used in the formula to determine depth of pit for each subject at the
3 WH. A larger WH coefficient resulted in an increased depth of pit and, thus, a higher WH (Figure 3).

\section{Measuring Devices}

The Trigno wireless sEMG system (Delsys, Boston, MA) was used to record upper limb and shoulder muscular activation of subjects during the work routine. Each sensor measured $37 \times 26 \times 15 \mathrm{~mm}$ and weighed $14 \mathrm{~g}$. Two bar electrodes and 2 reference bar electrodes were installed parallel within the sensor. One sensor was placed on each monitored muscle. Prior to sensor placement, the skin was prepared by shaving an area slightly bigger than the sensor and cleaning it with alcohol pads (70\%).

In total, 8 sensors were placed bilaterally on flexor carpi ulnaris, biceps brachii, deltoideus anterior, and 
upper trapezius, parallel to the muscle fibers according to the Seniam guidelines (Hermens et al., 1999). These muscles were selected due to their role during the attachment of the milking cluster. The cluster was held in the palm of the hand and, as such, requires wrist flexion, which is provided by the flexor carpi ulnaris. The biceps brachii is responsible for elbow flexion, which is relevant for lifting and holding of the cluster. The main function of the deltoideus anterior is shoulder abduction and flexion; thus, this muscle plays a vital role when the cluster is moved forward, away from the milker's body underneath the cow's udder. During this movement, the shoulder may also be lifted, which is facilitated by the upper trapezius.

Signal quality was checked before data collection using the EMGworks acquisition software (Delsys).
The software was further used to record the data with a standard sampling rate of $1,926 \mathrm{~Hz}$. At all times, an observer was present during the experiment. The observer documented the initiation of a new attachment procedure by swinging an accelerometer when the subject pressed the button to release the milking cluster. Therefore, an sEMG sensor was attached to the observer's hand and preset as an accelerometer with a standard sampling rate of $184.1 \mathrm{~Hz}$. As the sEMG and accelerometer data were recorded with the same software package, the accelerometer was used to mark the starting point of the attaching procedure in the sEMG data. The experimental procedures were filmed using Mobotix M15 conventional cameras (Mobotix AG, Langmeil, Germany) to be able to go back and evaluate ambiguous occurrences in sEMG data.

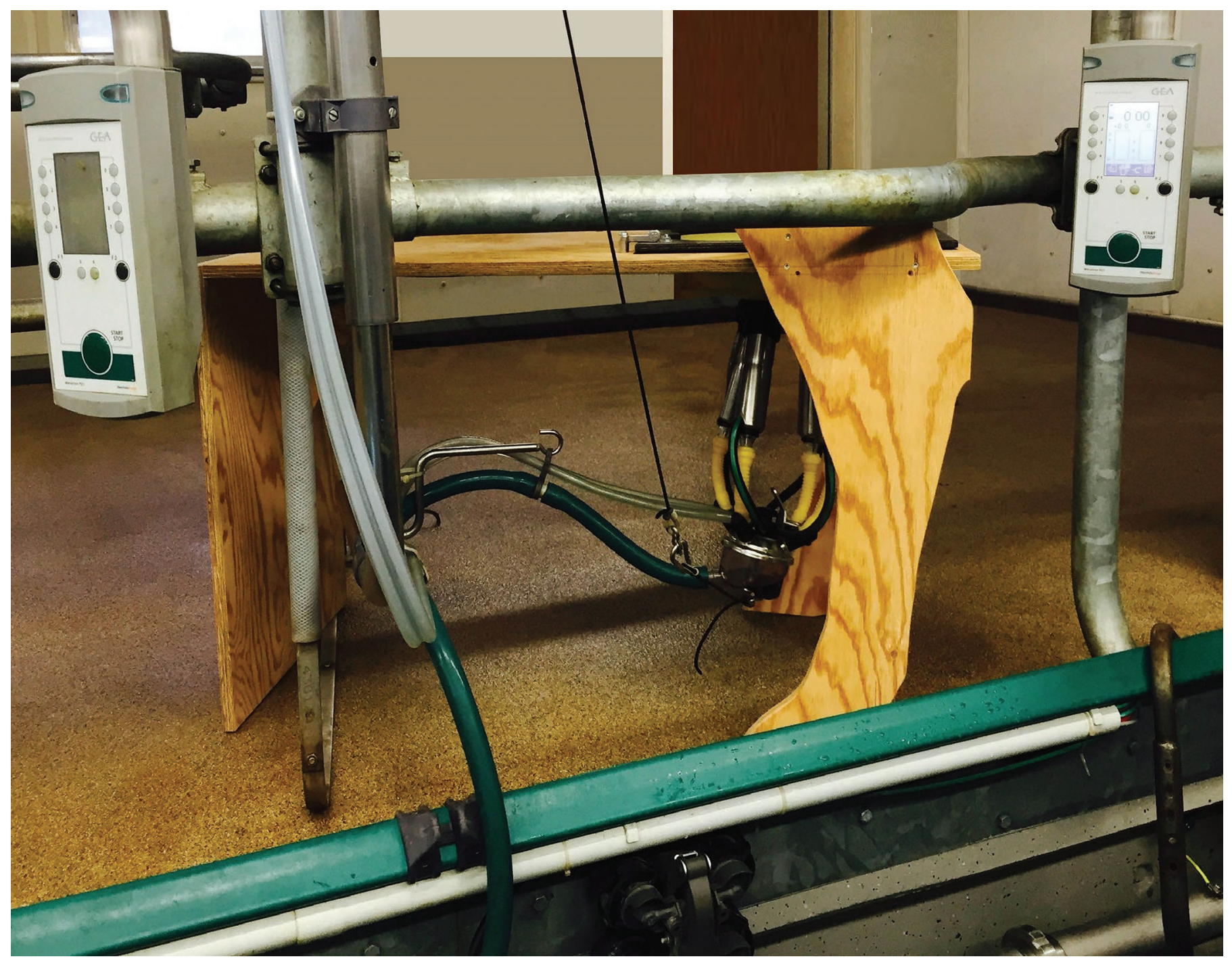

Figure 2. Artificial udder used in the experiment. Color version available online. 


\section{Experimental Procedure}

Data recordings took place between 0930 and 1230

h. The experimental procedure consisted of 3 elements.

1. $\boldsymbol{M V C}$. Each subject's recordings took place on the same day. Initially, the subject completed 2 unilateral MVC per monitored muscle (i.e., 16 $\mathrm{MVC}$ in total). The duration of each MVC was 3 to $5 \mathrm{~s}$ (Konrad, 2006) and the resistance was applied manually by the experimenter. The MVC of the flexor carpi ulnaris was performed by asking the subject to turn the ventral side of the lower arm upwards while the upper arm remained parallel to the trunk. The subject was then asked to flex the wrist upwards against a resistance with a joint angle at roughly $120^{\circ}$, whereby $180^{\circ}$ was a straight joint. The MVC of the biceps brachii was performed by asking the subject to perform an elbow flexion against resistance at approximately $90^{\circ}$. The MVC of the deltoideus anterior was performed by asking the subject to lift the lower and upper arm forward against a resistance while the elbow joint remained straight and the ventral side of the elbow and the radial side of the hand were facing upwards. The angle of the shoulder was hereby roughly at $150^{\circ}$, whereby $180^{\circ}$ was a straight joint. The MVC of the upper trapezius was performed by asking the subject to lift the shoulder upwards against the resistance while the elbow, shoulder, and wrist joints remained straight. If the maximal sEMG signal of the 2 contractions differed by more than $10 \%$, a third contraction was performed. The mean sEMG activity of these 2 contractions served as a reference for the experimental data and allowed for a comparison between and within subjects (see below). If a third contraction was performed, the mean was calculated from the 2 highest contractions.

2. Training the Attachment Technique. The subjects were instructed to attach the milking cluster with a defined technique to ensure that the attachment task was carried out similarly between the subjects. The arm that was furthest away from the cow's hind leg was always used to lift and hold the milking cluster, whereas the arm that was closest to the hind leg was used to attach the teat cups. Accordingly, the cluster was held in the left hand on the right side of the milking parlor and in the right hand on the left side of the milking parlor (holding), whereas the contralateral hand was used to attach the teat cups to the teat (attaching).

\section{Working Height}

low

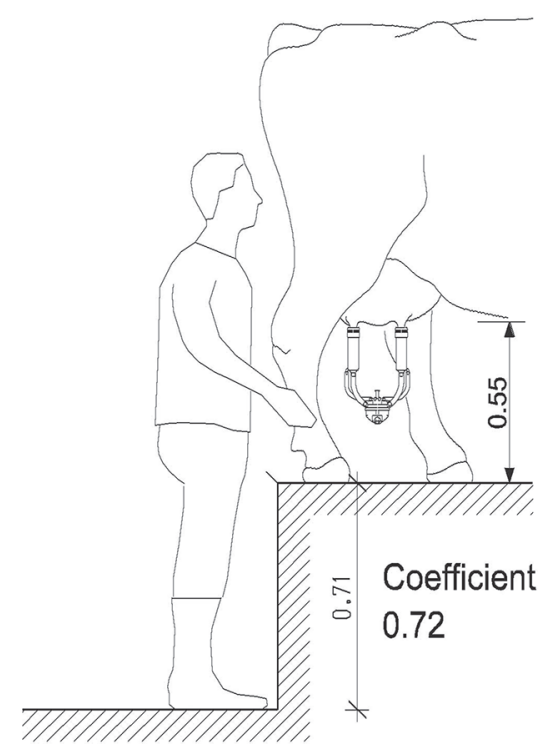

medium

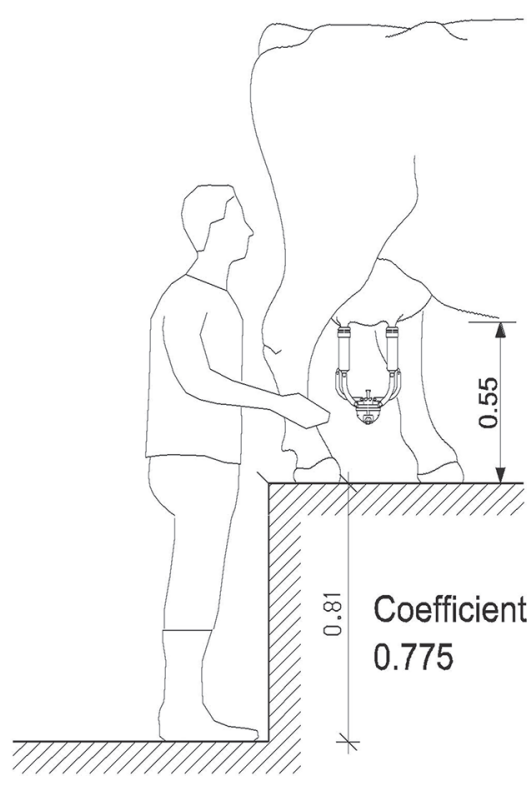

high

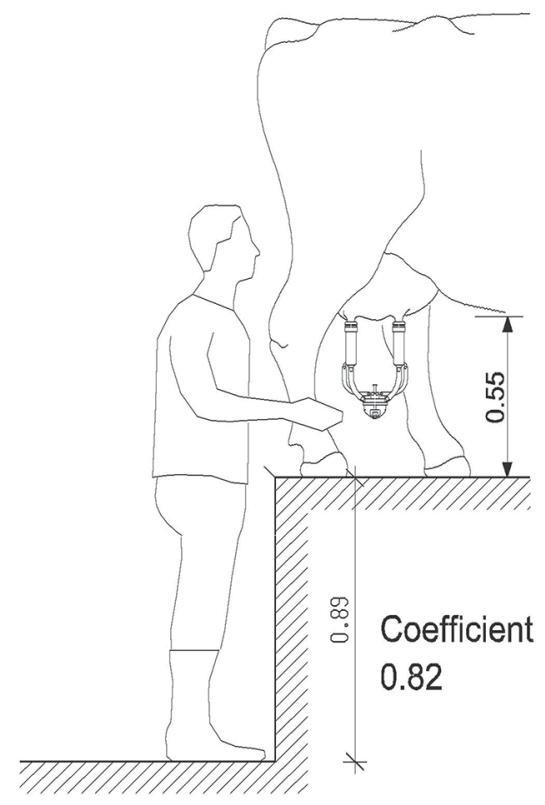

Figure 3. Three working height coefficients with a representative milker (body height of $1.75 \mathrm{~m}$ ) in a Herringbone $30^{\circ}$ parlor. Measurements in the figure are in meters. 
Initially, the cluster faced the floor and was held by a string, which was automatically released once the subject pressed the start button on the milking terminal. The subject was instructed to use the holding hand to swing the milking cluster and turn it around so that the teat cups faced upwards. Hereby the milk tubes were blocked and, therefore, air inlet was prevented due to the bending of the short milk tubes. The cluster was then shifted underneath the artificial udder (holding), and the teat cups were attached laterally in a U-shape (attaching), anterior to the artificial hind legs. The teat that was furthest away from the milker was hereby attached first. Subjects were instructed not to look at the teats while attaching the milking cluster. Instead, they were trained to hold the teat cup with their thumb and middle finger and use their index finger to feel the location of the teat and guide it into the cup (Figure 4).

Every subject was given 15 min to practice the milking cluster attachment in the instructed way before the recording period. The recording period began when the subjects confirmed that they felt comfortable and familiar with the attachment procedure.

3. Recording Period. Each subject was asked to attach the milking cluster 60 times. Each repetition started with the pressing of the start button, which released the milking cluster and was documented by the observer swinging the hand with the accelerometer. Each attachment procedure was followed by a 20 -s recovery phase. The time for cluster attachment was not limited. Five repetitions were carried out on each side of the milking parlor, with 1 min of recovery phase between sides. This procedure was then repeated once, resulting in 20 repetitions per $\mathrm{WH}$, with 10 repetitions on each side of the milking parlor. A new WH was installed after every 20 repetitions. The experimental setup was chosen to represent milking a group of 5 cows, which reflected milking in a practical setting. The WH as well as the parlor sides on which the data recording started were randomized between subjects. Six combinations of $\mathrm{WH}$ settings were therefore available. Each subject had a new combination of WH and started the milking on a new (right or left) side of the milking parlor.

\section{Data Analysis}

The data were processed in the software package EMGworks Analysis (Delsys). Both sEMG and ac-

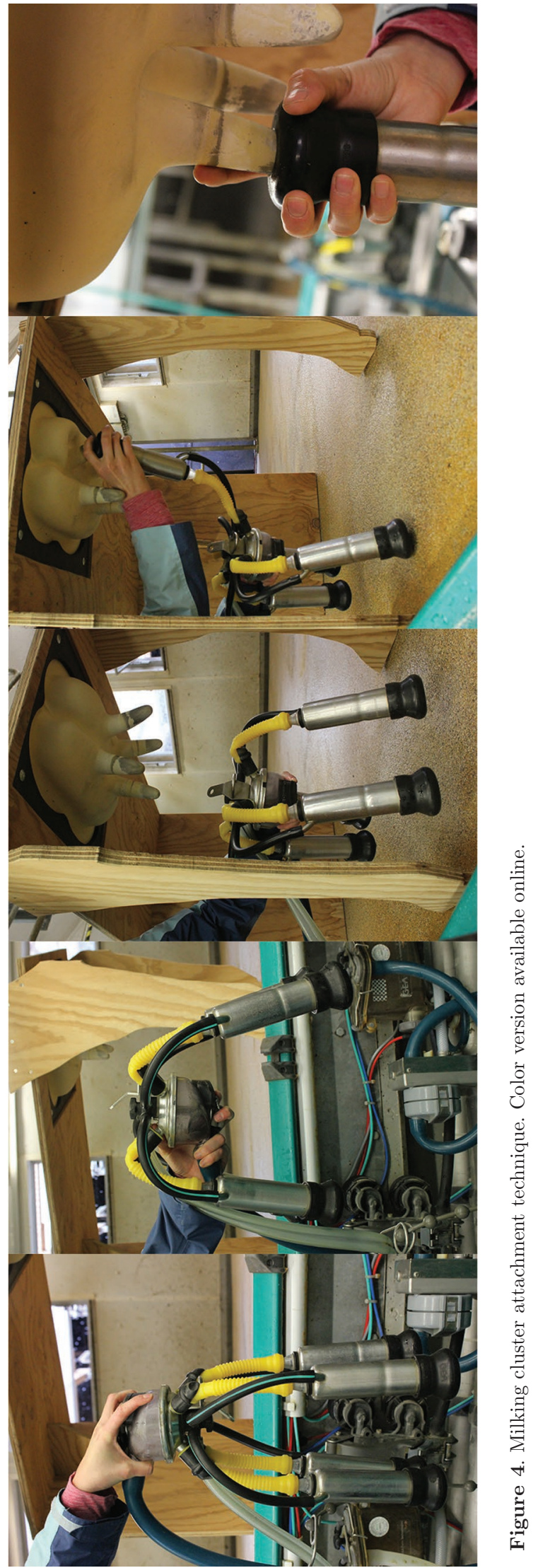

Journal of Dairy Science Vol. 100 No. 6, 2017 
celerometer data were processed simultaneously. Nonphysiological data, which can occur through movement of the sensors (for example, when they are caught on clothing or rails), appear as artifacts (peaks) in the raw sEMG data. Thus, the raw data were visually checked for artifacts, and attachment procedures with artifacts were omitted (sEMG sequences with artifacts were not evaluated) in the analyses. The percentage of omitted data was $1 \%$. Artifacts were defined as any visible shift $<5 \mathrm{~ms}$ with an increased amplitude based on the baseline sEMG. As the raw sEMG was measured in volts and, accordingly, had positive and negative values that equalize to zero, the root mean square values of the raw data were calculated with a window length of $0.25 \mathrm{~ms}$ (using one of the functions in the EMGworks analysis software). This resulted in roughly 481.25 sampling points, on average, to create positive values, which were then used for further analysis. Hereby, baseline noise was also removed, which ensured that all data of relaxed muscles originated from zero; no additional filters were used. We used $\mathrm{R}$ version 3.1.0 (R Core Team, 2013) to normalize the root mean square data to individual MVC of each subject's muscles by calculating the percentage of the mean MVC amplitude values. All sEMG data were subsequently reported as a percentage to provide mean contraction intensities.

The accelerometer traces consist of the $\mathrm{X}-, \mathrm{Y}-$, and Z-axes. The initial change before the peak of the acceleration traces (which was the result of the observer marking the beginning point) on the Z-axis was used as the starting point of the attachment procedure, whereas the ending point was established where the sEMG activity of the deltoideus anterior decreased almost to zero. The deltoideus anterior is responsible for lifting the arm forward or upward. When this muscle's activity decreases, the subject will not be able to hold the cluster underneath the udder, hence it was chosen as an ending point of the attaching procedure.

The time intervals between the starting and ending points were compared with the time intervals of the attachment procedure on the videos to ensure correct data alignment. The mean muscle contraction intensities during the attachment procedure were calculated with the software package EMGworks. This data set was then pasted into an Excel spreadsheet (Microsoft Office, Microsoft Corp., Redmond, WA). The data were lined up with the additional data representing $\mathrm{WH}$, subject height, side of the milking parlor, arm function, and repetition. Repetitions were coded from 1 to 5 . Hereby, 5 repetitions were carried out on 1 side of the milking parlor and represented the milking of one group of cows. These 5 repetitions were considered as a repetition group (12 in total, resulting in 60 attachment procedures). Files were separated into the arm hold- ing the milking cluster and the arm attaching the teat cups. Thus, for right and left muscles, 30 repetitions were evaluated for both the attaching and holding arm.

\section{Statistics}

Statistical analysis was performed separately for all 4 recorded muscles (on both sides) and 2 arm functions (holding and attaching functions of the right and left flexor carpi ulnaris, biceps brachii, deltoideus anterior, and upper trapezius) in $\mathrm{R}$ version 3.1.0, resulting in 16 models. A generalized linear mixed effects model ("Ime" method, Pinheiro and Bates, 2000) from the package "nlme" (Pinheiro et al., 2016) was fitted, where the mean contraction intensity was set as the outcome variable. The WH coefficient (factor with 3 levels), sex (factor with 2 levels), subject height (continuous), repetition (continuous), and their interactions were treated as fixed effects. Repetition group (6 repetition groups, each comprising 5 attachment procedures), nested in repetitions per $\mathrm{WH}$ (10 repetitions per $\mathrm{WH})$, nested in subject (30 repetitions per subject), were considered as random effects.

After fitting the model, the residuals were checked graphically for normal distribution and homogeneity of variance. To satisfy these assumptions, all mean contraction intensities were logit-transformed. In a few cases, it was necessary to remove outliers to ensure normally distributed residuals [outliers removed: 1 in the left biceps brachii (holding), 2 in the right flexor carpi ulnaris (attaching)].

The dredge function (package MuMin) was used to find the best model based on the smallest Bayesian information criterion and largest model weight (Bartoń, 2013). The model weight can be interpreted as the probability for a specific model to be optimal in the set of considered models given the data, where the model weight of all models in a set add up to 1 (Symonds and Moussalli, 2011). Here, our set included the maximum model as described above and all simpler models including the null model (with an intercept only; so-called allsubset approach). The evidence ratio reflects how many times the chosen model was more likely compared with the null model (Symonds and Moussalli, 2011). This approach in choosing a model is an alternative to frequentist $P$-value based testing; therefore, no $P$-values are presented.

\section{RESULTS}

The WH had no effect on contraction intensity of the flexor carpi ulnaris or the biceps brachii during either the holding or the attaching function. However, contraction intensity increased with WH and was highest 
at the high WH for both the holding and the attaching arm functions in the left and right deltoideus anterior and upper trapezius (Table 1; Figures 5 and 6). Only figures for the holding function of the right deltoideus anterior and the right upper trapezius were presented because the patterns we found were similar across the right and left sides, as well as the holding and attaching arm functions. Subject height had no effect on contraction intensity in any of the monitored muscles (Table 1). Sex had an effect on many of the monitored muscles; during the attaching arm function, muscular contraction intensities were higher in women than in men in the left and right flexor carpi ulnaris, the right biceps brachii, and the left and right deltoideus anterior (Table 2). This effect was also found in both left and right deltoideus anterior and upper trapezius for the holding arm function (Tables 1 and 2; Figures 5 and 6 ). Contraction intensity decreased with increasing repetition in the right flexor carpi ulnaris during the holding arm function. In addition, we found an interaction of sex and repetition in the right deltoideus anterior, where contraction intensity decreased over time in women but not in men (Figure 5). For the attaching arm, contraction intensity decreased over time in the left flexor carpi ulnaris, the left and right deltoideus anterior, and the right upper trapezius.

\section{DISCUSSION}

\section{Working Height}

The results of the current study showed that mean contraction intensities decreased bilaterally with decreasing WH in the deltoideus anterior, which is responsible for the forward or upward lifting of the arm, and the upper trapezius, which facilitates the lifting of the shoulder. For these muscles, contraction intensities were lowest when the milking cluster was attached with a low WH. This indicates that a lower $\mathrm{WH}$ may reduce physical strain of the shoulder muscles. No such effects were observed for the flexor carpi ulnaris or the biceps brachii, which are responsible for wrist flexion and elbow flexion, respectively. This finding shows that physical strain of the shoulder regions can be reduced by the correct usage of the milking health formula, which recommends lower WH. In praxis, this means WH can be fixed for one milkers' height only during or after the construction of the milking parlor, or individually for multiple milkers by using adjustable platforms. Whereas the present study only evaluated the formula in the herringbone parlor, Cockburn et al. (2015) developed a formula for the most common parlor types: rotary, parallel, herringbone $30^{\circ}$, herringbone $50^{\circ}$, and autotandem; their formula enabled the effective and

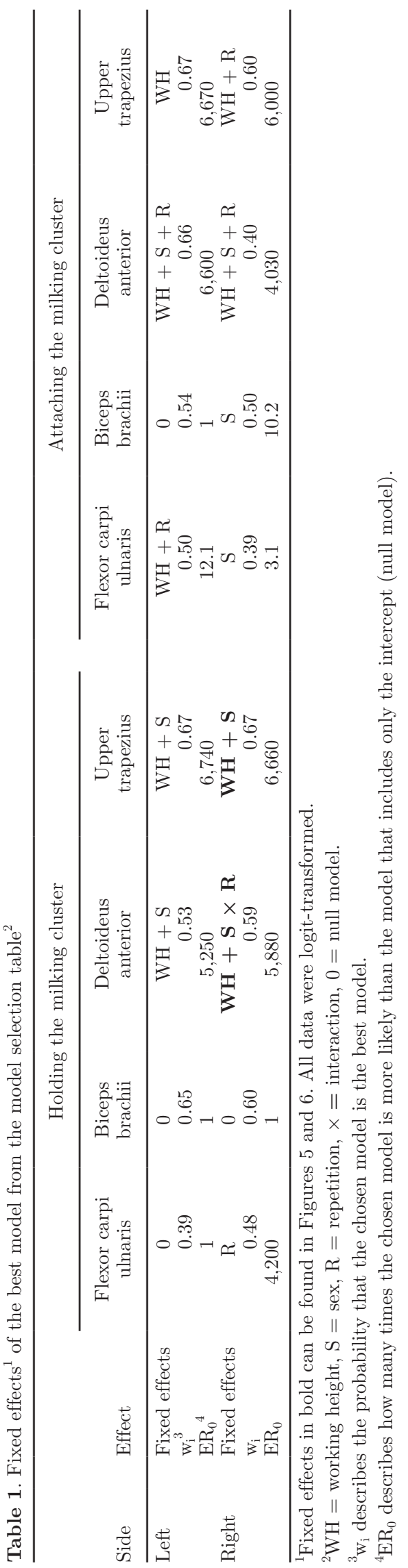

Journal of Dairy Science Vol. 100 No. 6, 2017 
Right Deltoideus Anterior - Holding

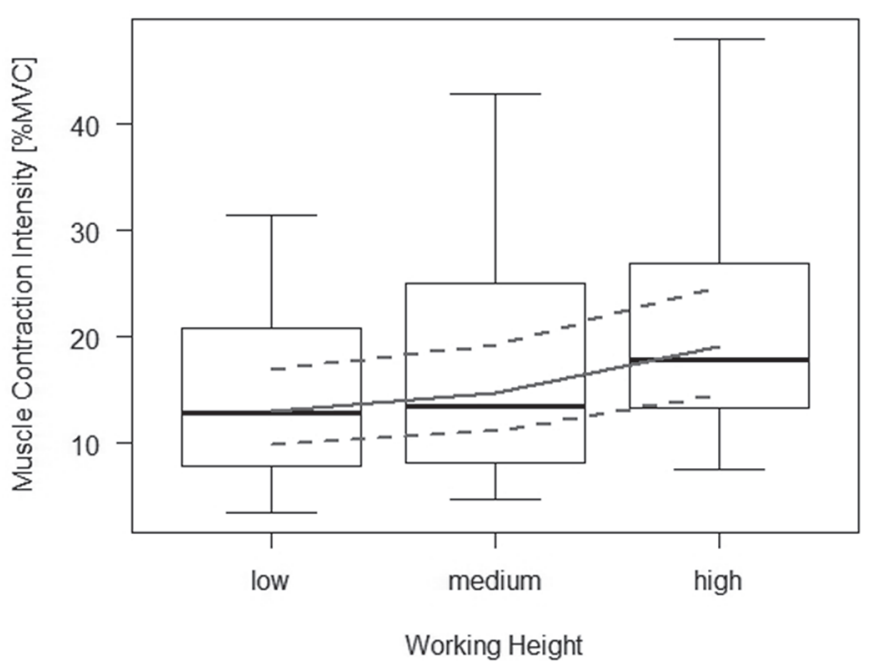

Right Deltoideus Anterior - Holding

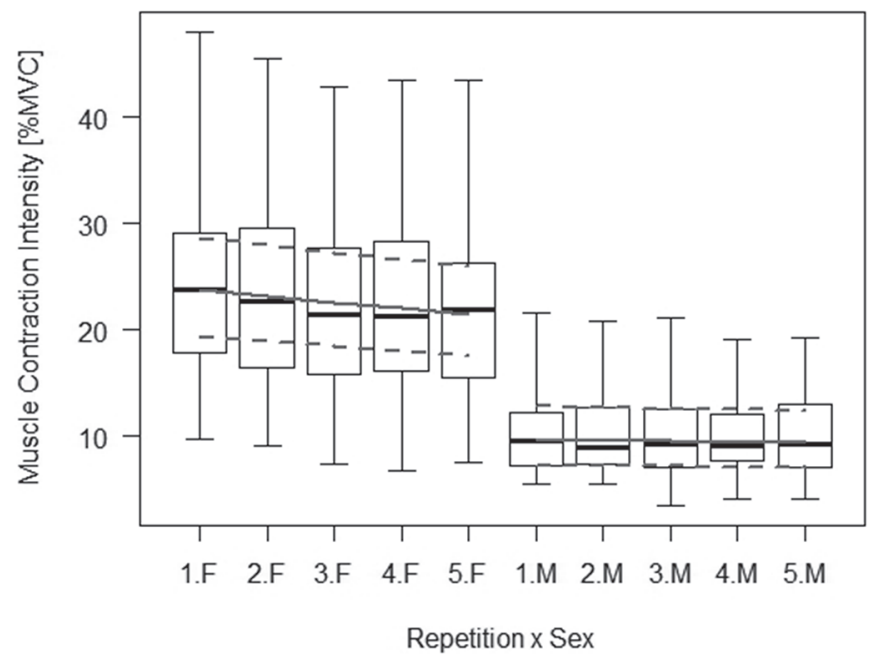

Figure 5. Contraction intensity of the right deltoideus anterior. Box plots show raw data. The thick black line indicates the median. The upper box indicates the 75 th percentile; the lower box indicates the 25 th percentile. The whiskers show the minimum and maximum. Lines show model prediction with upper and lower $95 \% \mathrm{CI} . \mathrm{F}=$ female; $\mathrm{M}=$ male. $\mathrm{MVC}=$ maximum voluntary contraction.

comparable setting of WH. The milking health formula hereby takes into account the milker's body height, and therefore accounts for the anthropometric characteristics of individuals. Although the muscular loads of these regions were lower than those of the flexor carpi ulnaris and the biceps brachii, milkers are primarily affected by shoulder problems, thus making it worthwhile to give these areas a closer consideration (Lunner Kolstrup and Jakob, 2016). However, the current study exclusively evaluated the effects in the herringbone $30^{\circ}$ parlor, so we cannot make statements regarding other milking parlor types.
Right Upper Trapezius - Holding

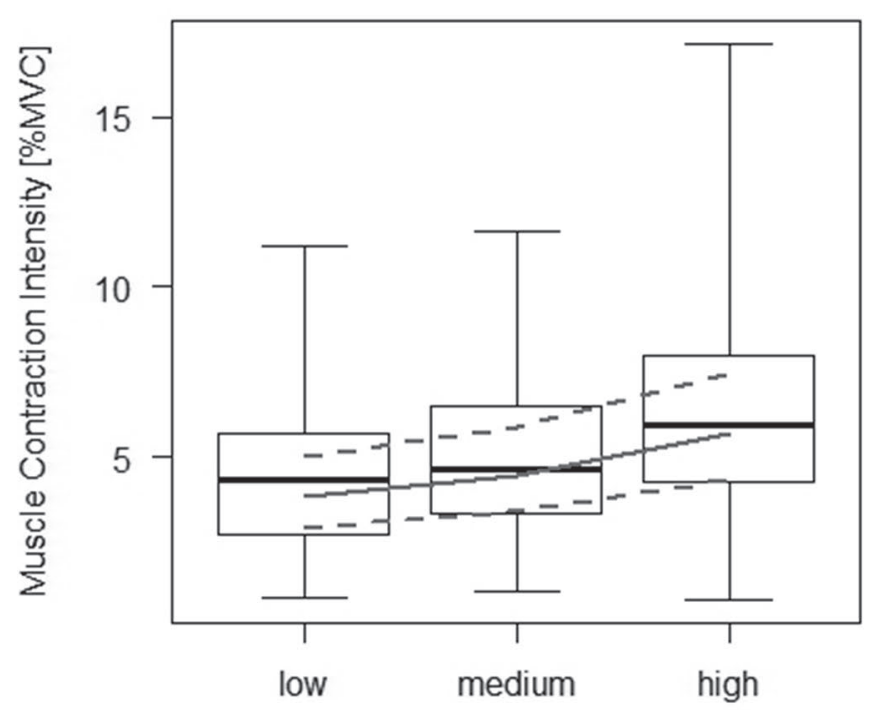

Working Height
Right Upper Trapezius - Holding

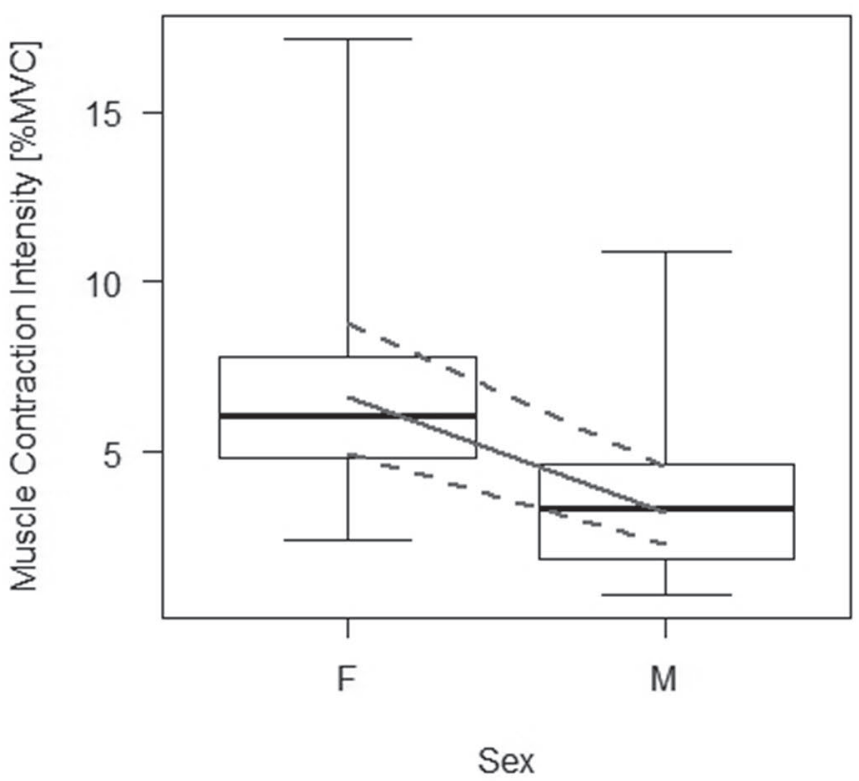

Figure 6. Contraction intensity of the right upper trapezius. Box plots show raw data. The thick black line indicates the median. The upper box indicates the 75 th percentile; the lower box indicates the 25 th percentile. The whiskers show the minimum and maximum. Lines show model prediction with upper and lower $95 \% \mathrm{CI} . \mathrm{F}=$ female; $\mathrm{M}=$ male. $\mathrm{MVC}=$ maximum voluntary contraction. 


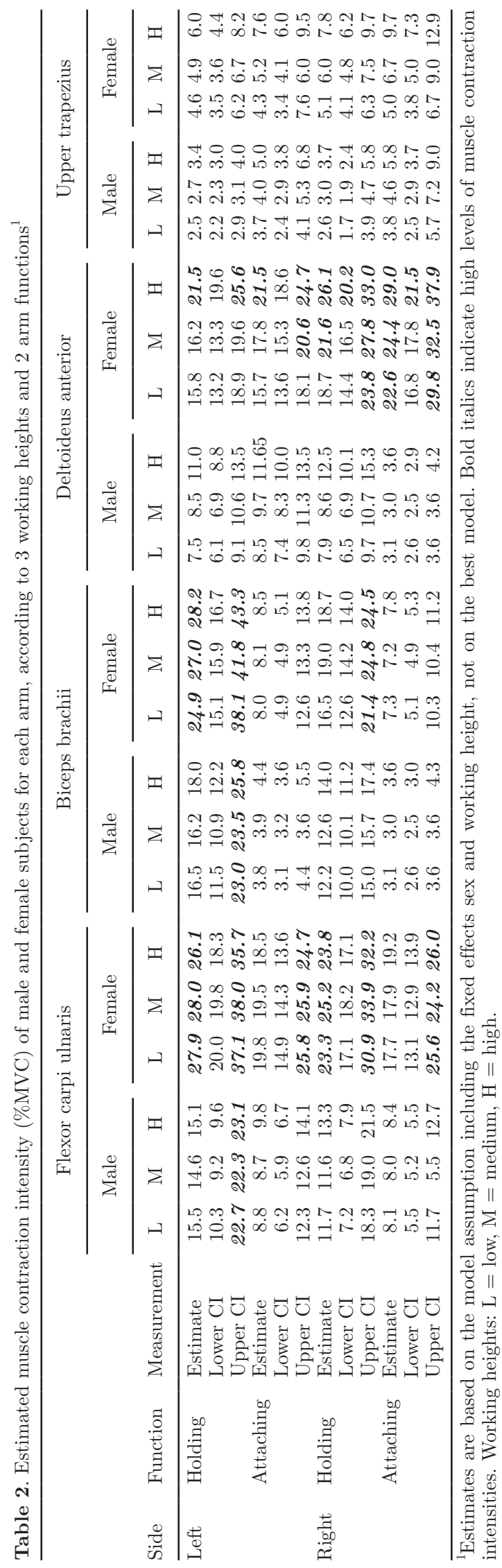

Whereas mean contraction intensities reported for the deltoideus anterior and the biceps brachii in the current study were relatively similar to those found in a previous study, the values for the upper trapezius were lower in our study (Liebers et al., 2009). However, previous research recommended WH at which the teat ends were at shoulder level, which was roughly equivalent to the high WH used in our study (Liebers et al., 2009; Jakob et al., 2012). Jakob et al. (2012) used an artificial udder that was fixed on a metal stand and shaped, but the proportions were not representative of a cow's leg. Their setup might have led to different postures or arm positions during cluster attachment, and therefore to different results than those found in our study, in which a true-to-scale hind leg held the artificial udder. This could be relevant when the milker is working at a lower WH at which the elbow and shoulder joints are more extended compared with a higher WH (Figure 3).

The current study evaluated $2 \mathrm{WH}$ at which the udder was below eye level (low and medium WH). This reduced contraction intensities, but visibly checking the udder became more difficult; as it was only possible if the milker tilted the head and neck. It would instead also be achievable for the milker to bend his knees. Subjects in the current study were instructed not to look at the teats while attaching the milking cluster, but, instead, to hold the teat cup with their thumb and middle finger and use their index finger to guide the teat into the cup. To reassure healthy cows, it is strongly recommended to examine the udder and teat condition before milking. However, the udder is visibly checked during the premilking and cleaning procedure, which is carried out before attaching the milking cluster. Although this task usually takes longer than the actual attaching of the cluster, it is carried out without a load and, thus, is associated with low physical stress. This agrees with former studies that found the attachment task to be most strenuous (Pinzke et al., 2001), which is why we focused particularly on this task in the current study. A recent work by Douphrate et al. (2013) further reported that $40 \%$ of herringbone parlor operators perceived attaching as the most difficult milking task versus $30 \%$ for parallel and $29 \%$ for rotary parlor operators. However, if milking parlors are built with a smaller depth of pit, and thus a lower $\mathrm{WH}$, solutions to enable udder visibility without a tilted head, such as camera or display solutions, could offer additional support in future milking parlors.

In the current study, muscular contraction intensities were higher in the holding arm than in the attaching arm. Stål et al. (1998) reported musculoskeletal disorders to be dominant in the hand used to hold the milking cluster. This supports the assumption that the high muscular contraction intensities during the hold- 
ing of the milking cluster may represent a risk factor for musculoskeletal disorders. The milking clusters used in the current study weighed $2.6 \mathrm{~kg}$, despite former studies reporting that lighter clusters $(1.4 \mathrm{~kg})$ positively affect ergonomics compared with heavy clusters $(2.6 \mathrm{~kg}$; Jakob et al., 2012). Although it has been stated that milking clusters commercially available range between 1.5 and $3.5 \mathrm{~kg}$ (Jakob et al., 2007), no representable data on average cluster weight is available to the best of our knowledge. Jakob et al. (2007) reported that torque ranged from $4.5 \mathrm{~N} \cdot \mathrm{m}$ with light clusters to almost 9 $\mathrm{N} \cdot \mathrm{m}$ with heavy clusters (Jakob et al., 2012). We chose to use these clusters because it was important to find differences between WH configurations, and a heavier cluster made it possible to show this effect more clearly. Mean contraction intensities in the current study were relatively high compared with Jakob et al. (2012). This may be because only subjects without milking routine were in our trial, as their muscles were not trained for the attaching procedure. It is likely that a lower level of practice results in higher muscle contraction intensities, despite a normalization to each subject's individual MVC.

\section{Sex}

An untrained subject requires a higher muscular activation to lift a load than a trained subject (Strasser et al., 2013). Similar explanations can be applied to the higher muscular activity in women as opposed to men. Strasser et al. (2013) described absolute maximum strength in men to be $30 \%$ higher than in women. This explains the sex-related findings of the current study, in which women presented higher muscular contraction intensities in all of the monitored muscles (Tables 1 and 2). Jonsson (1978) suggested limit values for muscular load, stating that the static load level "must not exceed $5 \%$ of MVC, the mean load level should not exceed $10 \%$ and must not exceed $14 \%$ of MVC." Hence, the muscular loads in our female milkers were considerably beyond the highest threshold, especially those of the flexor carpi ulnaris, biceps brachii, and deltoideus anterior (Table 2). Milking is a highly repetitive and forceful task that is carried out twice daily on family farms or for a period of 8 to $10 \mathrm{~h}$ in large herd operations and has previously been associated with awkward postures. Furthermore, female milkers have been reported to be at higher risk of developing musculoskeletal problems compared with nonmilking women (Stål et al., 1996). Concerning an ergonomic workplace design, it should be discussed if this work may be too strenuous for most women, especially as studies have previously reported a high prevalence of musculoskeletal disorders in female milkers (Lundquist et al., 2003; Stål et al., 1998, 2004).

\section{Repetition}

Increased efficiency, and thus more rapid movements, as well as high levels of repetition, have been linked to a higher potential of developing musculoskeletal disorders (NIOSH, 1997). We considered repetition in the statistical model primarily to account for information regarding the study design and to give a better estimation of the other variables. However, repetition had an effect in many of the best models, where mean contraction intensity decreased with increasing repetition number (Table 1). This finding agrees with a former study, which found a decrease in handling time with increasing repetition (Liebers et al., 2009). In our study, this effect was interacted with sex in the right deltoideus anterior in the attaching arm function, during which contraction intensities in women decreased with increasing repetition whereas those in men did not. This decrease may indicate that the females improved the efficacy of the movement and, therefore, reduced physical strain over the repetitions, which could be due to selecting subjects without a work routine. However, no kinematic analyses were conducted in the current study, which would have helped to detect potential change in the range of motion throughout the consecutive repetitions.

\section{Subject Height}

The milking health formula was created as a tool to calculate ideal depth of pits for subjects of varying body heights (Cockburn et al., 2015). Although considered in the statistical model, subject height had no effect on mean contraction intensities in any of the monitored muscles. Thus, we can conclude that the setting of depth of pits, under consideration of the subject height, offered an effective and objective way to compare WH between subjects with varying body heights. We can therefore consider the use of the milking health formula validated, yet should continue increasing the precision of the WH coefficients within the formula (Cockburn et al., 2015). Thus, the use of the milking health formula is valuable not only for farm practice but also for researchers, as it offers an objective and precise method for setting and comparing different WH.

\section{CONCLUSIONS}

The WH coefficient used in the milking health formula for the herringbone $30^{\circ}$ parlor should be medium or low to reduce contraction intensity of shoulder muscles. However, physical strain of the upper limbs does not seem to be affected by WH. Subject height had no effect on muscle contraction intensities. Future studies should use the milking health formula as a reference 
tool to improve comparability between studies. Mean contraction intensities were generally higher in women than in men; therefore, it is important for women to be aware of the high physical strain during milking.

\section{ACKNOWLEDGMENTS}

We thank the Swiss Farm Machinery Association (Bern, Switzerland) for funding this project. Further, we thank all the subjects for participating in the study, the farm staff for their support throughout the experiments, and Daniel Herzog (Agroscope, Ettenhausen, Switzerland) for creating Figures 1 to 4 .

\section{REFERENCES}

Bartoń, K. 2013. MuMIn: Multi-model inference, R package version 1.9.13. https://cran.r-project.org/web/packages/MuMIn/.

Cockburn, M., P. Savary, M. Kauke, M. Schick, U. Hoehne-Huckstadt, I. Hermanns, and R. Ellegast. 2015. Improving ergonomics in milking parlors: Empirical findings for optimal working heights in five milking parlor types. J. Dairy Sci. 98:966-974.

Douphrate, D. I., C. Lunner Kolstrup, M. Nonnenmann, M. Jakob, and S. Pinzke. 2013. Ergonomics in modern dairy practice: A review of current issues and research needs. J. Agromedicine 18:198-209.

Douphrate, D. I., D. Gimeno, M. W. Nonnenmann, R. Hagevoort, C. Rosas-Goulart, and J. C. Rosecrance. 2014. Prevalence of work-related musculoskeletal symptoms among US large-herd dairy parlor workers. Am. J. Ind. Med. 57:370-379.

Hermens, H. J., B. Freriks, R. Merletti, D. Stegeman, J. Blok, G. Rau, C. Disselhorst-Klug, and G. Hägg. 1999. European Recommendations for Surface Electromyography: Results of the SENIAM Project. Roessingh Research and Development b.v., Enschede, the Netherlands.

Jakob, M. 2010. Correlation between MSD among milking parlour operatives and specific work place design. Pages 289-292 in Ergonomics for All: Celebrating PPCOE's 20 Years of Excellence. CRC Press, Boca Raton, FL.

Jakob, M., F. Liebers, and S. Behrendt. 2012. The effects of working height and manipulated weights on subjective strain, body posture and muscular activity of milking parlor operatives-Laboratory study. Appl. Ergon. 43:753-761.

Jakob, M., S. Rose, and R. Brunsch. 2007. Einfluss der Melkstandauststattung auf die Arbeitsbelastung des Melkers. Z. Arbeitswiss. 61:173-181.

Jakob, M. C., F. Liebers, and S. Behrendt. 2009. The influence of working heights and weights of milking units on the body posture of female milking parlour operatives. Agric. Eng. Int. CIGR J. 11:1355.

Jakob, M. C., and M. Thinius. 2015. A strategy for workplace health promotion on German dairy farms. Agric. Eng. Int. CIGR J. $17: 173-180$.
Jonsson, B. 1978. Kinesiology: With special reference to electromyographic kinesiology. Electroencephalogr. Clin. Neurophysiol. Suppl. 1978:417-428.

Konrad, P. 2006. The ABC of EMG (Version 1.4). Noraxon, USA Inc., Scottsdale, AZ

Lange, W., and A. Windel. 2013. Kleine ergonomische Datensammlung. Vol. 15. Praxiswissen Arbeitssicherheit. Bundesanstalt für Arbeitsschutz und Arbeitsmedizin, Dortmund, Germany.

Liebers, F., M. Jakob, and S. Behrendt. 2009. Physical load during machine milking regarding weight of the milking unit and working level-Experimental study. Bornimer Agrartechnische Berichte 66:38-47.

Lundquist, P., M. Stäl, and S. Pinzke. 2003 Working conditions \& ergonomics when milking cows. Pages 59-65 in Fifth International Dairy Housing Proceedings of the 29-31 January 2003 Conference, Fort Worth, TX. ASABE, St. Joseph, MI. https://doi. org $/ 10.13031 / 2013.11603$

Lunner Kolstrup, C., and M. Jakob. 2016. Epidemiology of musculoskeletal symptoms among milkers and dairy farm characteristics in Sweden and Germany. J. Agromedicine 21:43-55.

NIOSH. 1997. Musculoskeletal disorders and workplace factors: A critical review of epidemiologic evidence for work-related musculoskeletal disorders of the neck, upper extremity, and low back. Department of Health and Human Services, National Institute of Occupational Safety and Health, Cincinnati, OH.

Patil, A., J. Rosecrance, D. Douphrate, and D. Gilkey. 2012. Prevalence of carpal tunnel syndrome among dairy workers. Am. J. Ind. Med. 55:127-135.

Pinheiro, J., D. Bates, S. DebRoy, D. Sarkar, and R Core Team. 2016. nlme: Linear and Nonlinear Mixed Effects Models. In R package version 3.1-127. Accessed Jun. 14, 2016. http://CRAN.R-project. org $/$ package $=$ nlme

Pinheiro, J. C., and D. M. Bates. 2000. Mixed-effects models in S and S-PLUS. Springer, New York, NY.

Pinzke, S., M. Stal, and G.-A. Hansson. 2001. Physical workload on upper extremities in various operations during machine milking. Ann. Agric. Environ. Med. 8:63-70.

R Core Team. 2013. R: A language and environment for statistical computing. R Foundation for Statistical Computing, Vienna, Austria. Vol. 2016, Accessed Jun. 14, 2016. http://www.R-project. org/.

Stål, M., C.-G. Hagert, and J.-E. Englund. 2004. Pronator syndrome: a retrospective study of median nerve entrapment at the elbow in female machine milkers. J. Agric. Saf. Health 10:247-256.

Stål, M., C.-G. Hagert, and U. Moritz. 1998. Upper extremity nerve involvement in Swedish female machine milkers. Am. J. Ind. Med. 33:551-559.

Stål, M., U. Moritz, B. Gustafsson, and B. Johnsson. 1996. Milking is a high-risk job for young females. Scand. J. Rehabil. Med. 28:95-104.

Strasser, H., B. Steinhilber, C. Anders, M. Jäger, H.-C. Scholle, N.-P Schumann, R. Seibt, and K. Kluth. 2013. Oberflächen-Elektromyographie in der Arbeitsmedizin, Arbeitsphysiologie und Arbeitswissenschaft. Z. Arbeitswiss. 67:113-127.

Symonds, M. R., and A. Moussalli. 2011. A brief guide to model selection, multimodel inference and model averaging in behavioural ecology using Akaike's information criterion. Behav. Ecol. Sociobiol. 65:13-21. 